\title{
Thoracic aorta vasoreactivity in rats under exhaustive exercise: effects of Lycium barbarum polysaccharides supplementation
}

\author{
Zhifang Zhao ${ }^{1 \dagger}$, Yan Luo ${ }^{1 \dagger}$, Guanghua $\mathrm{Li}^{1^{*}}$, Lingqin Zhu' ${ }^{1}$ Yin Wang ${ }^{1}$ and Xuehong Zhang ${ }^{2}$
}

\begin{abstract}
Background: Reduced arterial compliance is associated with an increased rate of morbidity and mortality in cardiovascular disease. Exercise is beneficial for compromised arterial compliance. However, the beneficial effects of exercise are lost with exhaustion. Lycium barbarum L. has been used in China for centuries to maintain good health. In this regard, the primary purpose of this study was to characterize the effects of the polysaccharides from Lycium barbarum (LBPs) on arterial compliance during exhaustive exercise.

Methods: A four-week swimming exercise program was designed for rats, and the blood levels of malondialdehyde (MDA), super oxide dismutase (SOD), nitric oxide(NO) and heat shock protein 70(HSP70) were detected. The tension of aorta rings was measured to evaluate the response of rats on noradrenaline (NA)-induced contractions.

Results: The rats administered LBPs showed longer swimming time until exhaustion than the control group of rats. Exercise-induced MDA elevation was repressed by LBPs supplementation. The LBPs-supplemented rats displayed a significant increase of SOD, NO, HSP70 than the non-supplemented rats. Additionally, LBPs significantly up-regulated the expression of eNOS and improved the endothelium-dependent vasodilatation of the aorta ring.
\end{abstract} Conclusion: Our study proved that LBPs administration significantly inhibited the oxidative stress, and improved the arterial compliance.

Keywords: Lycium barbarum polysaccharides, Exhaustive exercise, Thoracic aorta, Vasoreactivity

\section{Introduction}

Arterial compliance, the inverse of arterial stiffness, is now recognized as an important determinant of cardiovascular morbidity and mortality [1]. Exercise can affect arterial compliance. It is well known that aerobic exercise reduces arterial stiffness. Moderate-intensity aerobic exercise at $65 \%$ of its maximal oxygen uptake lowers both central and peripheral arterial stiffness [2]. In addition, twelve weeks of aerobic exercise enhances vascular compliance (especially of the arms and legs) in obese male adolescents [3]. However, the beneficial effects of exercise are lost with exhaustion. For example, Highintensity strength exercise leads to a decrease in arterial

\footnotetext{
*Correspondence: ghlee0404@163.com

${ }^{\dagger}$ Equal contributors

'Department of Physiology, School of Basic Medical Science, Ningxia Medical University, Yinchuan 750004, China

Full list of author information is available at the end of the article
}

compliance $[4,5]$. Twenty to forty hours of continuous mountain trail running decreases the large artery compliance [6]. Moreover, marathon runners have increased aortic stiffness compared to that of the control group [7]. In contrast, one-year of exercise fails to improve the arterial stiffness or function of heart failure with preserved ejection fraction (HFpEF) in patients [8]. The mechanism of different effects of exercise on arterial compliance remains unclear.

Lycium barbarum (also called Wolfberry, Fructus Lycii or Gouqizi), belonging to the plant family Solanaceae, has been widely used for 2000 years in traditional Chinese Medicine [9-11]. Polysaccharides (LBPs) which constitute more than $40 \%$ of the fruit extract are the major valuable and active ingredient in Lycium barbarum [12]. LBPs have been shown to exert a large variety of biological activities including eye-protective, anti-aging, antioxidant,

\section{Biomed Central}


immunoregulating, neuroprotective, cytoprotective and antitumor properties [13-17]. It has been reported that LBPs treatment prevented the increase of blood pressure in hypertension rats induced by the two-kidney, one clip method in vivo. LBPs-treated rats showed a significant decrease in the concentration of phenylephrine in isolated aortic rings as compared with non-treated hypertensive rats [18]. However, the effects of LBPs on arterial compliance in rats with exhaustive exercise have not been investigated. In the present study, we aimed to determine the effects of LBPs on the arterial compliance from lesions induced by exhaustive exercise.

\section{Materials and methods}

\section{Animals}

A total of 40 male Sprague Dawley rats $(180 \pm 20$ g) were bred, five per cage, in light-and temperature-controlled conditions (12 hours light: 12 hours dark; $24.0 \pm 0.2^{\circ} \mathrm{C}$ ) and provided with standard laboratory diet and tap water ad libitum. The experimental procedures were approved by the animal ethics committee of the Ningxia Medical University and Use Committee in accordance with the guidelines of the Council of the Physiological Society of China.

After an adaptation period of one week, all animals were randomly divided into 4 groups $(n=10)$ : control sedentary group (CS), swimming exercise group (SE), exhaustive swimming exercise group (ES), exhaustive swimming exercise with LBPs group (ES-LBP). The rats in ES-LBP group received $200 \mathrm{mg} / \mathrm{kg} /$ day by gavage for 28 days. In CS, SE, ES groups, the rats were given the same volume of isotonic saline solution by oral administration for 28 days. The dose of LBPs was chosen on the basis of preliminary experiments, which was safe and effective without undue toxicity in rats.

\section{Exercise protocol}

During the first week, rats were acclimated to swimming exercises for 5 days with increasing duration from 5 minutes on the first day to 60 minutes by the fifth day [19]. The rats in the control group were subjected to water immersion without exercises. The rats swam in a plastic tank (diameter, $60 \mathrm{~cm}$; depth, $80 \mathrm{~cm}$ ) filled with water at $32 \pm 1^{\circ} \mathrm{C}$. After acclimation, rats were assigned to swim for 60 minutes per day, 5 days per week, for 4 weeks (between 8:00 am and 12:00 am). At the end of the training, the rats of the ES and ES-LBP groups were subjected to a swim to exhaustion with a load of $5 \%$ of their body weight strapped on their backs. The point of exhaustion was defined when a rat failed to rise to the surface of water, drown over 10 seconds and could not maintain coordination [20]. This exhaustion time was subsequently recorded.

\section{Samples collection}

All animals were anesthetized with urethane $(1.5 \mathrm{~g} / \mathrm{kg})$ and sacrificed immediately after the exhaustive exercise. The chest was rapidly opened and the thoracic aorta was carefully isolated in order to preserve the vascular endothelium, which was then placed into modified cold Krebs' solution. The isolated vessel was cut into rings of approximately 3-4 $\mathrm{mm}$ wide for measuring isometric force. The rest of the aorta was frozen in liquid nitrogen immediately and stored at $-80^{\circ} \mathrm{C}$ for the assay of endothelial NO synthase (eNOS) mRNA expression . Blood was collected from inferior vena cava in heparinized tube and centrifuged at $1,700 \times \mathrm{g}$ for 10 minutes (at $4^{\circ} \mathrm{C}$ ) to obtain plasma. The plasma was frozen at $-80^{\circ} \mathrm{C}$ to measure the expression of malondialdehyde (MDA), super oxide dismutase (SOD), nitric oxide (NO) and heat shock protein 70(HSP70).

\section{Assay of isometric force in Rat aorta rings}

The isolated aortic rings were cleaned to remove the adherent tissues and hung in 10-ml organ bath with Krebs' solution at $37^{\circ} \mathrm{C}, \mathrm{pH} \mathrm{7.4}$, and containing $95 \% \mathrm{O}_{2}$ and $5 \%$ $\mathrm{CO}_{2}$. The modified Krebs' solution was composed of the following components: $110 \mathrm{mM} \mathrm{NaCl}, 4.6 \mathrm{mM} \mathrm{KCl}$,

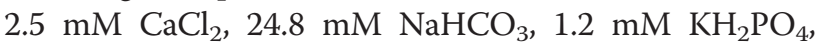
$1.2 \mathrm{mM} \mathrm{MgSO}_{4}$, and 5.6 g glucose. The tissue's isometric tension was measured with force transducers that connected with a BL- $420 \mathrm{E}^{+}$biological function experimental system (Chengdu Technology and Market, Chengdu, China). The vessel rings were equilibrated for 1 hour with the tension of $2.0 \mathrm{~g}$ and pre-contracted with $\mathrm{KCl}$ (60 $\mathrm{mM})$ to produce the maximal KCL-induced contractile plateau. Subsequently the cumulative doseresponse curve for noradrenaline (NA) $\left(10^{-10}-10^{-5} \mathrm{M}\right)$ was obtained. The values of the NA-induced contraction were expressed as a percentage of maximal contraction induced by $\mathrm{KCl}$.

\section{Measurement of SOD, MDA and nitrite/nitrate (NOx) levels in plasma}

The oxidative stress indices were measured to explore whether LBP could reduce exhaustive exercise-induced oxidative stress. The levels of SOD, MDA and NOx $\left(\mathrm{NO}^{2-}\right.$ and $\left.\mathrm{NO}^{3-}\right)$ were determined by using commercially available kits (Nanjing Jiancheng Bioengineering Institute, Nanjing, China) according to the manufacturer's instructions.

\section{HSP70 determination}

The plasma level of HSP70 was detected by a commercially available ELISA kit (Cusabio Biotechnology, Wuhan, China). The amount of HSP70 in plasma was estimated from the calibration curve ranging from 62.5 to $4000 \mathrm{pg} / \mathrm{ml}$. 
Table 1 GenBank accession code, primer sequences, and predicted size of the amplified product

\begin{tabular}{lll}
\hline Gene & Primer sequences & GenBank \\
\hline eNOS & Forward primer: 5'-CACACTGCTAGAGGTGCTGGAA-3' & NM_021838 \\
& Reverse primer: 5'-TGCTGAGCTGACAGAGTAGTAC-3' & \\
\multirow{3}{*}{-actin } & Forward primer: 5'-TCATGAAGTGTGACGTTGACATCCGT-3' & \\
& Reverse primer: 5'-CCTAGAAGCATTGGGGTGCAGGATG-3' & \\
\hline
\end{tabular}

\section{RT-PCR analysis}

Total RNA was prepared from the thoracic aorta using RNA AxyPrep Pure RNA isolation kit (AXYGEN, USA) according to the manufacturer's instructions. The purity and concentration of RNA was determined by spectrophotometry at $260 \mathrm{~nm}$ and $280 \mathrm{~nm}$. Complementary DNA (cDNA) was synthesized using a reverse transcription kit (TransGen Biotechnology, Beijing). Quantitative PCR was performed using a quantitect SYBR green PCR kit (TransGen Biotechnology, Beijing) as follows: 35 cycles of denaturation at $94^{\circ} \mathrm{C}$ for $30 \mathrm{sec}$, annealing at $62^{\circ} \mathrm{C}$ for $30 \mathrm{sec}$ and extension at $72^{\circ} \mathrm{C}$ for $30 \mathrm{sec}$. Primers used for the PCR were shown in Table 1. Relative gene expression levels were determined using the $2^{-\Delta \mathrm{Ct}}$ method.

\section{Statistical analysis}

Results were presented as the mean \pm SD. Two-way ANOVA was used to evaluate any differences between the two sets of dose-response curves. The remaining data were evaluated by one-way ANOVA and Student's t-test. The statistical analyses were performed by SPSS for Windows 11.5.0 software. $\mathrm{P}<0.05$ was considered to indicate a statistically significant difference.

\section{Results}

\section{Contractile response of vascular ring to NA}

Vascular dysfunction is related to increased vasoconstriction and weakened diastolic function. Therefore, we are interested in determining whether there is any change in the vascular function by detecting the vascular reactivity of aortic rings to a physiological modulator, noradrenaline (NA). Cumulatively added NA $\left(10^{-10}-10^{-5} \mathrm{M}\right)$ caused concentration-dependent contractile responses in isolated aortic rings. We found that there was no significant difference between the SE and the CS group, while the ES group significantly increased the vasoconstrictive response to NA $(P<0.01)$, LBPs treatment decreased the vasoconstrictive effect $(P<0.01)$ (Figure 1$)$. Furthermore, the contractile responsiveness to NA of the SE group was significantly lower than that of the ES $(P<0.01)$ and ES-LBP $(P<0.01)$ groups (Figure 1$).$

\section{Effects of LBPs on body weight and exhaustive exercise} time in rats

After four weeks of swimming exercise, no significant difference was observed in body weight in either group
(Table 2). However, as shown in Figure 2, LBPs prolonged the swimming time of rats compared with the ES group $(P<0.05)$, which was $77.07 \%$ higher.

\section{Effects of LBPs on biochemical parameters after exhaustive exercise}

It is well known that SOD can inhibit the oxidation of oxyamine by the xanthine-xanthine oxidase system. Therefore we evaluated the plasmic level of SOD. As shown in Figure 3a, the SOD level in the ES-LBP, SE groups significantly increased compared with that in the CS group $(P<0.05$ and $P<0.01$ respectively). However, the plasmic SOD level of exhaustive swimming rats was significantly lower than that of the ES-LBP and SE rats $(P<0.01)$. The results demonstrated that LBPs were able to increase antioxidant enzyme activities to attenuate the oxidative stress induced by exhaustive exercise.

Exhaustive exercise induces the generation of free radicals which may cause an increase in lipid peroxidation [21]. Measuring MDA is one of the most widely used approaches for evaluating oxidative damage to lipids. Figure $3 \mathrm{~b}$ illustrates that the plasmic MDA levels of SE or ES-LBP rats significantly decreased compared with that of ES rats $(P<0.05$ and $P<0.01$ respectively). This result indicates that LBPs can attenuate lipid peroxidation.

$\mathrm{NO}$ is an important vasodiator factor produced by vascular endothelial cells. We found that there was a significant increase in the SE group. As expected, the NO level

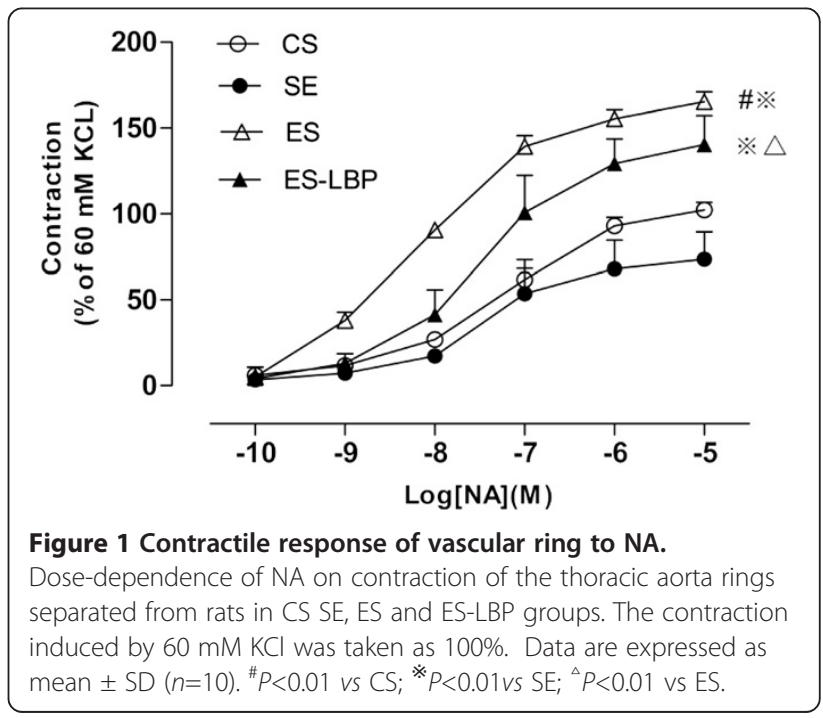


Table 2 Effects of LBP on body weight in rats

\begin{tabular}{llllll}
\hline Group & Before experiment & One week & Two week & Three week & Four week \\
\hline CS & $191.67 \pm 26.90$ & $204.83 \pm 13.43$ & $264.08 \pm 12.31$ & $304.44 \pm 9.97$ & $346.58 \pm 15.55$ \\
SE & $187.5 \pm 4.74$ & $209.53 \pm 6.15$ & $258.43 \pm 9.88$ & $309.35 \pm 19.11$ & $340.5 \pm 22.31$ \\
ES & $191.2 \pm 10.77$ & $210.67 \pm 10.91$ & $263.5 \pm 14.05$ & $304.58 \pm 17.12$ & $329.13 \pm 15.06$ \\
ES-LBP & $198.2 \pm 9.66$ & $215.14 \pm 7.22$ & $267.70 \pm 6.96$ & $312.08 \pm 10.14$ & $344.33 \pm 14.91$ \\
\hline
\end{tabular}

Effects of LBPs on body weight in rats. The values are expressed as mean \pm SD $(n=10)$.

was significantly reduced by exhaustive exercise. Further, we found this reduction induced by exhaustive exercise could be reversed by LBPs treatment (Figure 3c).

The expression of heat shock proteins (HSPs) is induced by hyperthermia ischemia, oxidative cytokine, muscular stress, glucose deprivation, alterations in calcium and $\mathrm{pH}$ [22]. HSP70 is a group of binding proteins with molecular weight of $70 \mathrm{KD}$, which is significantly increased by high-intensity exercise [23]. To determine the expression of HSP70 after exercise and supplement with LBPs, the plasmic level of HSP70, analyzed by ELISA, showed an immediate increase after both exercise sessions. As shown in Figure 3d, the HSP70 levels of SE or ES rats were increased. Furthermore, LBPs treatment induced a much higher increase in the ES group $(P<0.01)$.

\section{Expression of eNOS mRNA}

As the NO level can be up-regulated by LBPs, we therefore examined the effect of LBPs on the expression of eNOS in the aorta after exhaustive exercise. The expression of eNOS mRNA in aorta of four groups was shown in Figure 4. There were significant differences in the eNOS mRNA expression level among different groups. The eNOS expression was increased in both SE and ESLBP groups $(P<0.01)$. However, the level of eNOS expression was significantly attenuated in rats after exhaustive exercise $(P<0.01)$. LBPs treatment significantly reversed the inhibition of the eNOS expression in rats from ES group $(p<0.01)$.

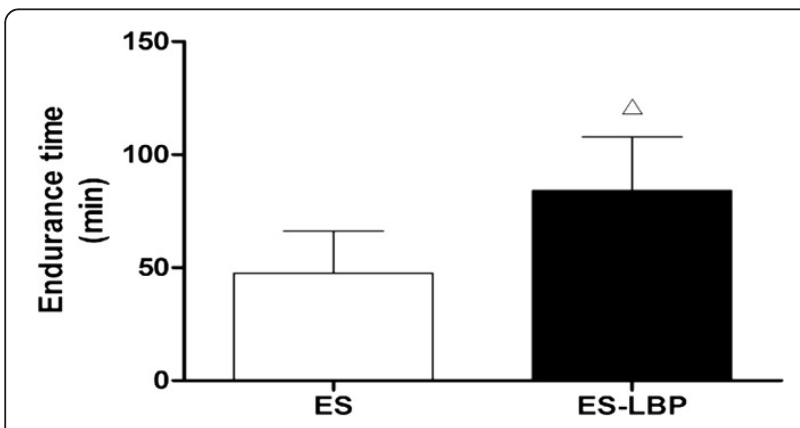

Figure 2 Effects of LBPs on exhaustive exercise time in the rats. LBPs supplementation significantly increased the time to fatigue compared to that of the ES. Data are mean \pm SD $(n=10)$. ${ }^{\triangle} P<0.01$ vs ES.

\section{Discussion}

The effects of LBPs on vascular vasoreactivity in exhaustive exercise rats were investigated. The major finding of this study was that the contraction induced by NA in thoracic aorta was increased in the presence of exhaustive exercise. Furthermore, supplementation with the LBPs for 4 weeks remarkably improved the vascular reactivity of ES-LBP rats compared to the ES rats (Figure 1). As the arterial compliance is judged by the responsiveness to $\mathrm{NA}$, the results showed that the compliance or distensibility of aorta was increased in LBPs treated animals [24].

Arterial compliance, the inverse of arterial stiffness, is now recognized as an important determinant of cardiovascular morbidity and mortality [1], which can increase the workload placed on the myocardium. The changes in arterial compliance of exercise training rats depend on the exercise mode, intensity and duration. Twelve weeks of air board exercise leads to an increase in cardio-respiratory fitness and vascular compliance, which may reduce the risk of later development of cardiovascular disease [3] and improve coronary artery perfusion preventing ischemic events [25], and decline pulse pressure and wall stress [26]. Moreover, Nickel [27] showed that 30 minutes of moderate-intensity aerobic exercise transiently increased small arterial compliance after exercise, but not sustained. Extremely high volume exercise may be associated with decreases in cardiovascular function and large artery compliance [6]. Ahmadi et al. [28] recently reported that coronary artery calcification was associated with impaired aortic compliance.

The present study has confirmed these varying effects of exercise on arterial compliance. In SE rats, which were subjected to swimming exercise for four weeks, the attenuated contractile responses of aorta to NA were clearly observed, whereas in rats exposed to exhaustive swimming exercise, depressed vasodilator response was observed (Figure 1). This inhibition was completely reversed by the treatment of LBPs in the ES group. In isolated aortic rings of LBPs-treated rats, the responsiveness to phenylephrine was attenuated in comparison with non-treated hypertensive rats [18].

Generally, exhaustive exercise induced oxidative stress impaired endothelial function [29] that decreased artery 

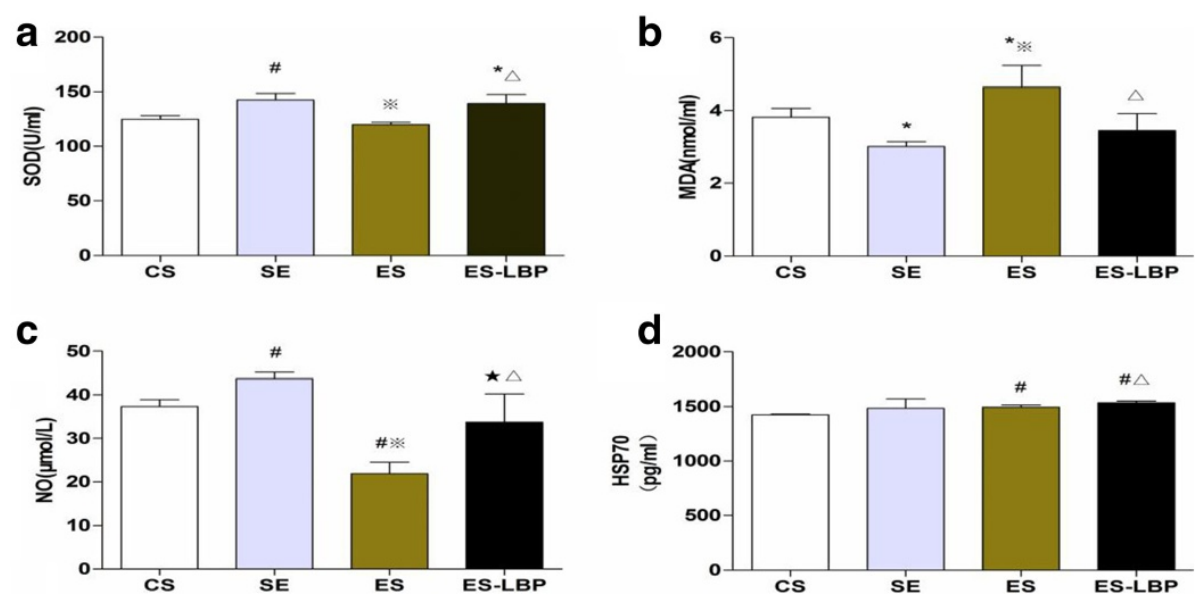

Figure 3 Effects of LBPs supplement and exhaustive exercise on SOD (a), MDA (b), NO (c) and HSP70 (d) expression in the rats. Values are expressed as mean \pm SD $(n=10) .{ }^{*} P<0.05$ and ${ }^{\#} P<0.01$ vs $C S ;{ }^{\star} P<0.05$ and ${ }^{*}<0.01$ vs SE; ${ }^{\wedge} P<0.01$ vs ES.

compliance [30], which may interfere with NA-dependent vasoconstriction. The present study indicated that a bout of exhaustive swimming exercise caused a significant increase in oxidative stress, which decreased the serum antioxidant enzyme SOD and increased the lipid hydroperoxides MDA. LBPs were shown to be effective in avoiding oxidative stress and cleaning out the excess free radical and decreasing the level of lipid peroxidation $[10,31,32]$. These increases in super oxide levels were correlated with attenuated responsiveness to NA. Our previous study also showed that LBPs could enhance the immune function in exhausted swimming rat [33]. Combination with results of this study, LBPs is a useful protective agent in rats of exhaustive exercise, and whether LBPs are helpful for athletes needs a further research to confirm.

$\mathrm{NO}$, derived from a biochemical reaction catalyzed by eNOS [34], plays an important role in the regulation of

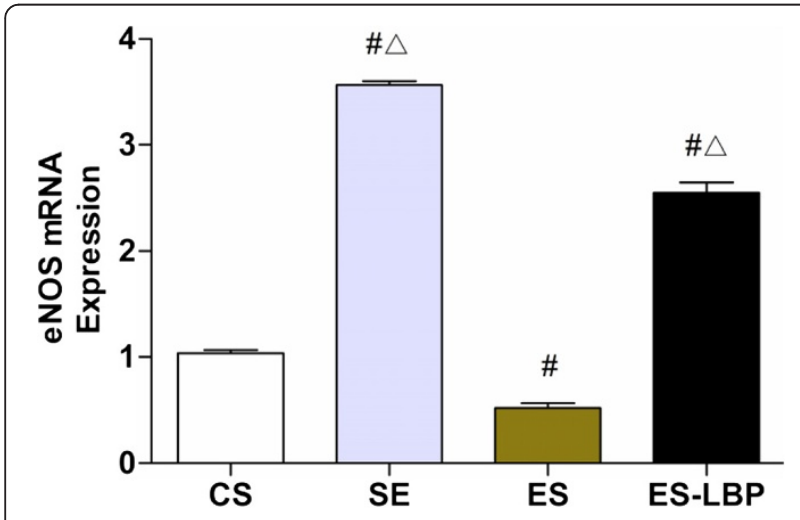

Figure 4 Effects of LBPs on eNOS mRNA expression in thoracic aorta separated from rats in different groups. Values are expressed as mean \pm SD $(n=10) .{ }^{\#} P<0.01$ vs $C S ;{ }^{\Delta} P<0.01$ vs ES. vascular tension [35]. The most important activity of NO may be vasodilation in the cardiovascular system, which is usually used as a surrogate index of endothelial function [35]. Studies have demonstrated that arterial stiffness was regulated by the endothelium through the release of NO [36]. Our data showed that LBPs could enhance the expression of eNOS, elevate NO levels and consequently inhibit the contractile response to NA. Previous studies have suggested that $\mathrm{N}$-nitro-L-arginine methyl ester increased the contraction to phenylephrine in the aortic rings of LBPs-treated rats in vitro. LBPs reduced the phenylephrine-induced contraction which may be mediated by increasing the production of endothelium-derived relaxation factor (EDRF) [18]. In addition, aortic contractility of LBPs-treated rats reduced due to attenuated responsiveness to NA and probably to increase in plasmic level of NO. The up-regulation of SOD levels during exercise training might lead to improvement in endothelial function through an increase in NO production [37].

Heat shock proteins (HSP) belong to the family of stress-responsive proteins that are induced by oxidative stress, which are essential for modulating cell function and maintaining protein homeostasis [38,39]. As a stress protein, the response of HSP70 is different according to the intensity and form of movement, which provides new ideas and methods to further understand the campaign laws and institute more scientific physical training and exercise training [40,41]. In ES-LBP, the HSP70 levels were significantly increased compared with that of ES. Meanwhile, the attenuation of the NA-induced aortic contraction was observed in ES-LBP rats. Thus, HSP70 may take part in this attenuation through protecting the cells from the deleterious effects of ROS and reducing oxidative stress. 


\section{Conclusion}

In conclusion, this study clearly indicates that the contractile response to NA is attenuated by LBPs treatment in ES-LBP rats. The exhaustive swim time is also prolonged by LBPs supplement through activation of the antioxidant defense system. Meanwhile, LBPs can upregulate the expression of eNOS, NO and HSP70. However, the mechanism of blunted contractile response to NA in aorta of LBPs-treated rats is not fully investigated in this study, further research including molecular study is required to investigate this mechanism.

\section{Competing interests}

The authors declare that they have no competing interests.

\section{Authors' contributions}

GL: dissertation guidance, interpretation of the data and and drafted the manuscript; ZZ: randomization of the protocol training of animals, literature review; YL: molecular biology assays; LZ: ELISA assays assistance and biochemical assays; YW: paper revise; XZ: animal training assistance; All authors read and approved the final manuscript.

\section{Acknowledgements}

This study was supported by National Natural Science Foundation of China, No. 81060230, 81050352 and Ningxia Natural Science Foundation No. NZ10111, NZ13055. The authors also wish to thank Jason Zhang for English assistance.

\section{Author details}

'Department of Physiology, School of Basic Medical Science, Ningxia Medical University, Yinchuan 750004, China. ${ }^{2}$ Department of Pediatrics, General Hospital of Ningxia Medical University, Yinchuan 750004, China.

\section{Received: 3 May 2013 Accepted: 17 October 2013}

Published: 24 October 2013

\section{References}

1. Cavalcante JL, Lima JAC, Redheuil A, et al: Aortic stiffness current understanding and future directions. I Am Coll Cardiol 2011, 57(14):1511-1522.

2. Heffernan K, Collier S, Kelly E, et al: Arterial stiffness and baroreflex sensitivity following bouts of aerobic and resistance exercise. Int I Sports Med 2007, 28(3):197.

3. Song JK, Stebbins CL, Kim TK, et al: Effects of 12 weeks of aerobic exercise on body composition and vascular compliance in obese boys. J Sports Med Phys Fitness 2012, 52(5):522-529.

4. Otsuki T, Maeda S, lemitsu M, et al: Vascular endothelium-derived factors and arterial stiffness in strength-and endurance-trained men. American Physiol-Heart Circ Physiol 2007, 292(2):H786-H791.

5. Kawano $\mathrm{H}$, Tanimoto $\mathrm{M}$, Yamamoto $\mathrm{K}$, et al: Resistance training in men is associated with increased arterial stiffness and blood pressure but does not adversely affect endothelial function as measured by arterial reactivity to the cold pressor test. Exp Physiol 2008, 93(2):296-302.

6. Burr J, Bredin SS, Phillips A, et al: Systemic arterial compliance following ultra-marathon. Int J Sports Med 2012, 33(03):224-229.

7. Vlachopoulos C, Kardara D, Anastasakis A, et al: Arterial stiffness and wave reflections in marathon runners. Am J Hypertens 2010, 23(9):974-979.

8. Fujimoto N, Prasad A, Hastings JL, et al: Cardiovascular effects of 1 year of progressive endurance exercise training in patients with heart failure with preserved ejection fraction. Am Heart J 2012, 164(6):869-877.

9. Deli MA, Yang D, Li S-Y, et al: Lycium barbarum extracts protect the brain from blood-brain barrier disruption and cerebral edema in experimental stroke. Plos One 2012, 7(3):e33596.

10. Shan XZ, Zhou JL, Ma T, et al: Lycium barbarum polysaccharides reduce exercise-induced oxidative stress. Int J Mol Sci 2011, 12(2):1081-1088.

11. Potterat OG: (Lycium barbarumandL. chinense): phytochemistry, pharmacology and safety in the perspective of traditional uses and recent popularity. Planta Med 2009, 76(01):7-19.
12. Chang RC-C, So K-F: Use of anti-aging herbal medicine, lycium barbarum, against aging-associated diseases. What do we know so far? Cell Mol Neurobio/ 2007, 28(5):643-652.

13. Ho YS, Yu MS, Yik SY, et al: Polysaccharides from wolfberry antagonizes glutamate excitotoxicity in rat cortical neurons. Cell Mol Neurobiol 2009, 29(8):1233-1244.

14. Wu HT, He XJ, Hong YK, et al: Chemical characterization of Lycium barbarum polysaccharides and its inhibition against liver oxidative injury of high-fat mice. Int J Biol Macromol 2010, 46(5):540.

15. Tang W-M, Chan E, Kwok C-Y, et al: A review of the anticancer and immunomodulatory effects of Lycium barbarum fruit. Inflammopharmacology 2012, 20(6):14-307.

16. Chang HM, But PPH, Yao SC: Pharmacology and applications of Chinese materia medica. Singapore: World Scientific Publishing Company Incorporated; 2001.

17. Potterat O: Phytochemistry, pharmacology and safety in the perspective of traditional uses and recent popularity. Planta Med 2010, 76:7-19.

18. Jia YX, Dong JW, Wu XX, et al: The effect of lycium barbarum polysaccharide on vascular tension in two-kidney, one clip model of hypertension. Sheng Li Xue Bao 1998, 50(3):309-314.

19. Sampaio-Barros M, Farias-Silva E, Grassi-Kassisse D, et al: Effect of swimming session duration and repetition on metabolic markers in rats. Stress: Int J Biol Stress 2003, 6(2):127-132.

20. Thomas D, Marshall K: Effects of repeated exhaustive exercise on myocardial subcellular membrane structures. Int J Sports Med 1988, 9(4):257-260.

21. Skarpańska-Stejnborn A, Basta P, Pilaczyńska-Szcześniak $Ł$, et al: Black grape extract supplementation attenuates blood oxidative stress in response to acute exercise. Biol Sport 2010, 27(1):41-46.

22. Fehrenbach $\mathrm{E}$, Northoff $\mathrm{H}$ : Free radicals, exercise, apoptosis, and heat shock proteins. Exerc Immunol Rev 2001, 7:66-89.

23. Noble EG, Moraska A, Mazzeo RS, Roth DA, Olsson MC, Moore RL, Fleshner M: Differential expression of stress proteins in rat myocardium after free wheel or treadmill run training. J App/ Physiol 1999, 86(5):1696-701.

24. Dunbar SL, Tamhidi L, Berkowitz DE, et al: Hindlimb unweighting affects rat vascular capacitance function. American J Physiol-Heart Circ Physiol 2001, 281(3):H1170-H1177.

25. Kass DA, Saeki A, Tunin RS, et al: Adverse influence of systemic vascular stiffening on cardiac dysfunction and adaptation to acute coronary occlusion. Circulation 1996, 93(8):1533-1541.

26. Belz GG: Elastic properties and Windkessel function of the human aorta. Cardiovasc Drugs Ther 1995, 9(1):73-83.

27. Nickel KJ, Acree LS, Gardner AW: Effects of a single bout of exercise on arterial compliance in older adults. Angiology 2011, 62(1):33-37.

28. Ahmadi N, Nabavi V, Hajsadeghi F, et al: Impaired aortic distensibility measured by computed tomography is associated with the severity of coronary artery disease. Int J Cardiovasc Imaging (formerly Cardiac Imaging) 2011, 27(3):459-469.

29. Knez WL, Coombes JS, Jenkins DG: Ultra-endurance exercise and oxidative damage: implications for cardiovascular health. Sports Med 2006, 36(5):429-441.

30. Wilkinson IB, MacCallum $\mathrm{H}$, Cockcroft JR, et al: Inhibition of basal nitric oxide synthesis increases aortic augmentation index and pulse wave velocity in vivo. Br J Clin Pharmacol 2002, 53(2):189-192.

31. Niu A-J, Wu J-M, Yu D-H, et al: Protective effect of Lycium barbarum polysaccharides on oxidative damage in skeletal muscle of exhaustive exercise rats. Int J Biol Macromol 2008, 42(5):447-449.

32. Duan CB, Sun ZJ: Supplementation of Lycium barbarum polysaccharides protection of skeletal muscle from exercise-induced oxidant stress in mice. African J Pharm Pharmacol 2012, 6(9):643-647.

33. Jing $H$, Hong-peng $L$, Zhou $X$, Guang-hua L: The effect of LBP on immune function of exhausted swimming exercise in mice. J Liaoning University of TCM 2009, 11(8):234-236

34. Li GH, Katakura M, Maruyama M, et al: Changes of noradrenaline-induced contractility and gene expression in aorta of rats acclimated to heat in two different modes. Eur J App/ Physio/ 2008, 104(1):29-40.

35. Maiorana A, O'Driscoll G, Taylor R, et al: Exercise and the nitric oxide vasodilator system. Sports Med 2003, 33(14):1013-1035.

36. Bellien J, Favre J, lacob M, et al: Arterial stiffness is regulated by nitric oxide and endothelium-derived hyperpolarizing factor during changes in blood flow in humans. Hypertension 2010, 55(3):674-680. 
37. Higashi Y, Yoshizumi M: Exercise and endothelial function: role of endothelium-derived nitric oxide and oxidative stress in healthy subjects and hypertensive patients. Pharmacol Ther 2004, 102(1):87-96.

38. Asea A: Hsp70: a chaperokine. In Novartis Foundation symposium; 2008 Volume 1999. Chichester; New York: John Wiley; 2008:173.

39. Atalay M, Oksala N, Lappalainen J, et al: Heat shock proteins in diabetes and wound healing. Curr Protein Pept Sci 2009, 10(1):85.

40. Banfi $G$, Dolci $A$, Verna $R$, et al: Exercise raises serum heat-shock protein 70 (Hsp70) levels. Clin Chem Lab Med 2004, 42(12):1445-1446.

41. Guixia C, Junwei B: Progress of the research on the effect of exercises on HSP70 expression in cardiac and skeletal muscles. J Jilin Institute of Phys Educ 2010, 26(5):83-85.

doi:10.1186/1550-2783-10-47

Cite this article as: Zhao et al.: Thoracic aorta vasoreactivity in rats

under exhaustive exercise: effects of Lycium barbarum polysaccharides supplementation. Journal of the International Society of Sports Nutrition 2013 10:47.

\section{Submit your next manuscript to BioMed Central and take full advantage of:}

- Convenient online submission

- Thorough peer review

- No space constraints or color figure charges

- Immediate publication on acceptance

- Inclusion in PubMed, CAS, Scopus and Google Scholar

- Research which is freely available for redistribution 